\title{
Combining Ability for Yield and Quality Traits in Early Generation Inbred Lines of Okra
}

\author{
V. Harshavardhan Gowda, Shantappa Tirakannanavar*, R.C. Jagadeesha, \\ V.D. Gasti, S.M. Veeresha and Ashok
}

Department of Biotechnology and Crop Improvement, University of Horticultural Sciences Bagalkot, Karnataka, India

*Corresponding author

K e y w or d s
Combining ability,
Line x tester
analysis, Yield and
quality parameters,
okra

Keywords

Combining ability, Line $\mathrm{x}$ tester analysis, Yield and quality parameters Article Info

Accepted: Available Online: 10 July 2018

\section{A B S T R A C T}

An experiment was conducted at Department of Biotechnology and Crop Improvement, Kittur Rani Channamma College of Horticulture, Arabhavi during the year 2013-14. Ninety-four hybrids derived by crossing fortyseven lines with two testers were evaluated along with parents and commercial check in Latin square design. Data was been subjected to line $\mathrm{x}$ tester analysis. Variance due to crosses was significant for all the yield and quality parameters studied. The maximum and significant gca effects in desirable direction was observed in the Line-44 for total fruit yield per plant, Line-7 for average fruit weight, Line-46 for number of fruits per plant, Line-14 for fruit length, Line-14 for fruit diameter. The crosses Line24 x Arka Anamika (201.496) followed by Line -23 X IC550848 (176.180) were identified as good specific combiners for total fruit yield. The present investigation reveals that the parents can be used to exploit heterosis.

\section{Introduction}

Okra [Abelmoschus esculentus (L.) Moench] is a fast growing annual is commonly known as bhendi or lady's finger in India. India is the largest producer of okra in the world with an annual production of 63.46 million tonnes from an area of 0.532 million hectares with a productivity of 11.9 tonnes per hectare (Anon., 2014). It occupies fifth position, in area among the vegetables grown in India and is a tremendous export potential as fresh vegetable, which accounts for 60 per cent of the fresh vegetables exported from India (Jasol, 1989).

Although India is the leading country in okra production, but the productivity is very low due to poor yielding varieties, hence there is a scope for hybrids. The concept of combining 
ability is landmark in the hybridization programme. Combining ability analysis is one of the effective approaches available for estimating the combining ability effects that helps in selecting desirable parents and crosses for the exploitation of heterosis. In order to identify potential cross combinations, it is very important to screen out the parent materials for genetic diversity and combining ability (Kumar and Kumar, 2015). Line $\mathrm{x}$ Tester method is considered one of the effective ways for estimating the general and specific combining ability to select the inbred lines for further generation (Kempthorne, 1957). The present investigation was carried out to evaluate early generation inbred lines for combining ability.

\section{Materials and Methods}

The investigation on evaluation of early generation inbred lines and hybrid seed production in okra was undertaken during the year 2013-2014 in Kharif season. The experimental material comprised of 47 early generation inbred lines which were selected based on their per se performance for yield and quality attributes, 2 testers and their 94 F1 hybrids along with one commercial check. Each of the 47 lines was crossed with each of the two testers (Arka Anamika and IC550848) to derive $94 \mathrm{~F} 1$ hybrids following Line $\mathrm{x}$ Tester method (Kempthorne, 1957).

These 94 hybrids along with 49 parents and one commercial check were sown at spacing of $60 \mathrm{x} 30 \mathrm{~cm}$ apart. Observations were recorded on the tagged five plants chosen at random in each genotype and in each replication. The mean of five plants was taken for analysis. Combining ability analysis was carried out according to the formulae given by Kempthorne (1957) through computergenerated program, WINDOSTAT (edition 9.1)

\section{Results and Discussion}

The analysis of variance showed significant variation among the crosses and line $\mathrm{x}$ tester for all seven characters, indicating the variation among the crosses may be due to positive interaction between male and female for expression of characters, further it reveals the possibility of non additive gene action, this can be exploited by recurrent selection or heterotic breeding (Table 1). However the mean sum of squares among lines and testers where non-significant, but lines and tester have interacted in a positive way to create variability in the cross combinations. The nonsignificant variation in lines indicates that they may have derived from same gene pool; these results are confirmatory with Laxman et al., (2013) and Khatik et al., (2012).

Critical evaluation of the results with respect to specific combining ability effects, out of 94 crosses, five crosses have shown high sca for the yield parameters. Crosses Line-3 x AA (L $x$ L), Line-12 x AA (L x L), Line-26 x IC (A x $\mathrm{H})$, Line-31 x IC ( $\mathrm{H} \times \mathrm{H})$ and Line-33 x IC $(\mathrm{H}$ $\mathrm{x} H$ ) have high sca for over all yield parameters (Table 3). Indicating significant sca may be due to the involving the parents $\mathrm{H}$ x H, L x H, L x H, A x H and L x L. The sca involving $\mathrm{H} \times \mathrm{H}$ combination could be used in developing of the varieties to exploit additive gene action by pedigree. Whereas, the crosses involving the combination of $\mathrm{L} \times \mathrm{L}$ could be used for exploitation of heterosis by recurrent selection (Table 3). For yield parameters combining ability was reported by Nagesh et al., (2014), Hazem et al., (2013) and Ashwani et al., (2013). Crosses Line-6 x IC ( $\mathrm{H} \times \mathrm{H})$, Line-10 x IC $(\mathrm{H} \times \mathrm{H})$, Line-20 x AA (L x L), Line- $24 \times$ IC $(\mathrm{A} \times \mathrm{H})$ and Line-45 $x$ IC (A x $\mathrm{H})$ have high sca for over all fruit quality. It indicates significant sca may be due to the involving of the parents $\mathrm{H} \times \mathrm{H}, \mathrm{L} \times \mathrm{H}, \mathrm{L} \times \mathrm{H}$ and $\mathrm{L} x \mathrm{~L}$. 
Table.1 Analysis of variance (mean sum of squares) of line x tester analysis for various characters in okra

\begin{tabular}{|c|c|c|c|c|c|c|c|}
\hline Character & Replication & Crosses & Lines & Tester & Line $*$ Tester & Error & Total \\
\hline Degree of freedom & 1 & 93 & 46 & 1 & 46 & 93 & 187 \\
\hline Number of fruits & 0.211 & $24.690 * *$ & 28.578 & 26.138 & $20.770 * *$ & 0.417 & 12.488 \\
\hline Average fruit weight (g) & 0.562 & $22.719 * *$ & 13.823 & $779.943 * *$ & $15.153 * *$ & 0.635 & 11.617 \\
\hline $\begin{array}{l}\text { Total fruit yield per plant } \\
\text { (g) }\end{array}$ & 717.860 & $28792.16 * *$ & 25063.01 & $646092.5 * *$ & $19101.75 * *$ & 663.556 & 14652.94 \\
\hline Fruit length (cm) & $2.960 *$ & $7.518 * *$ & 7.365 & 9.165 & $7.635 * *$ & 0.463 & 3.985 \\
\hline Fruit diameter (mm) & 1.577 & $6.586 * *$ & 6.324 & 8.924 & $6.796 * *$ & 1.029 & 3.795 \\
\hline No. of ridges on fruit & 0.005 & $4.120 * *$ & 2.629 & $121.282 * *$ & $3.065 * *$ & 0.005 & 2.052 \\
\hline No. of locules per fruit & 0.005 & $3.899 * *$ & 2.443 & $118.090 * *$ & $2.873 * *$ & 0.005 & 1.942 \\
\hline
\end{tabular}


Table. 2 General combining ability effects for growth parameters in okra

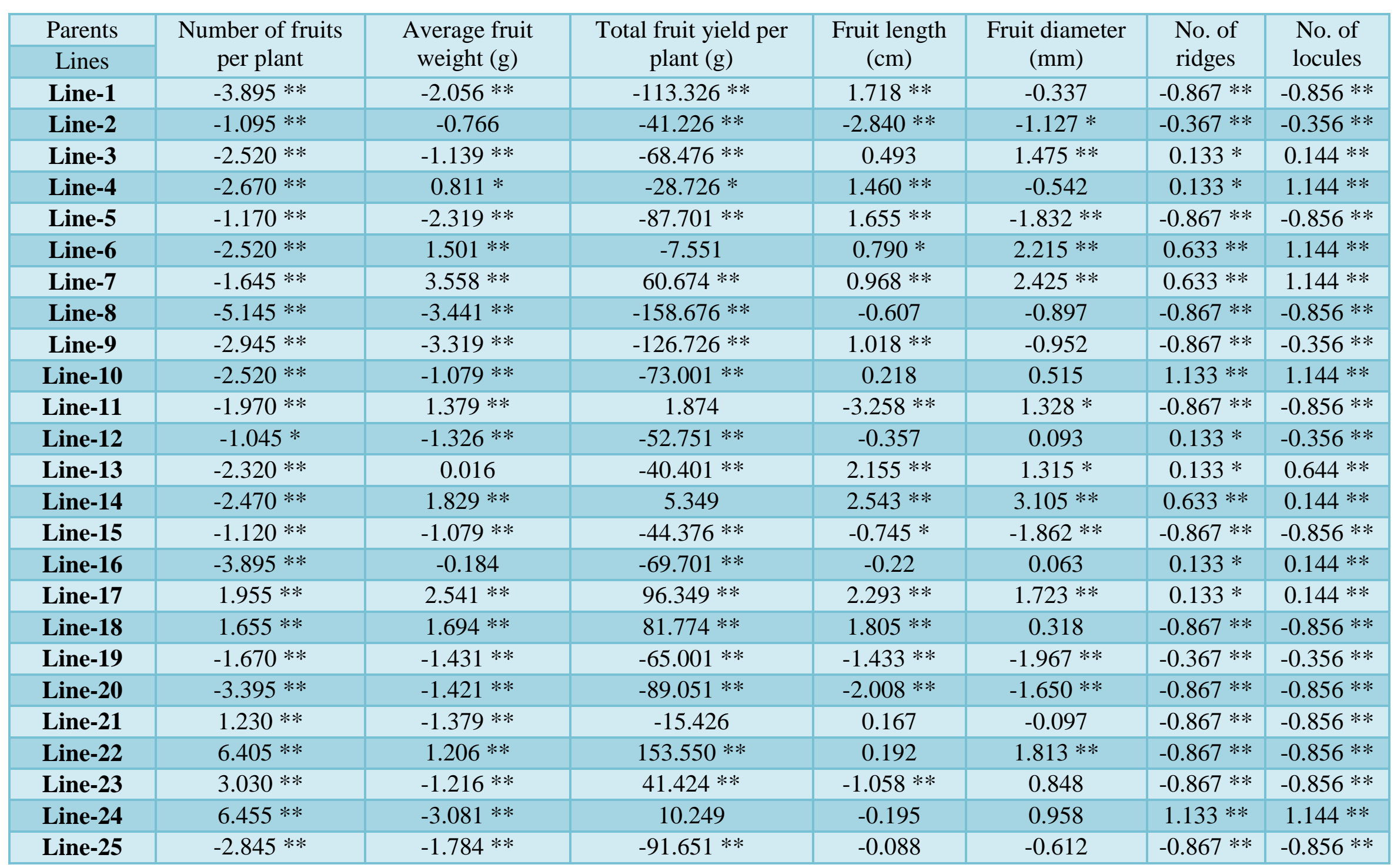




\begin{tabular}{|c|c|c|c|c|c|c|c|}
\hline Line-26 & $2.330 * *$ & $-2.366 * *$ & -11.301 & $1.673 * *$ & -0.897 & $-0.367 * *$ & $0.144 * *$ \\
\hline Line-27 & $-1.895 * *$ & 0.436 & $-28.201 *$ & 0.143 & $1.690 * *$ & $0.633 * *$ & $0.644 * *$ \\
\hline Line-28 & $-1.670 * *$ & $2.644 * *$ & $41.749 * *$ & -0.508 & -0.325 & $-0.867 * *$ & $-0.856 * *$ \\
\hline Line-29 & $-4.695 * *$ & 0.219 & $-73.601 * *$ & $-3.383 * *$ & -0.647 & $1.133 * *$ & $1.144 * *$ \\
\hline Line-30 & $1.155 * *$ & $-3.269 * *$ & $-64.101 * *$ & -0.712 & 0.048 & $-0.867 * *$ & $-0.856 * *$ \\
\hline Line-31 & $1.230 * *$ & $3.221 * *$ & $116.025 * *$ & -0.508 & $-1.415 *$ & $0.133 *$ & $0.144 * *$ \\
\hline Line-32 & $1.105 * *$ & -0.544 & 2.074 & -0.143 & -0.072 & $0.133 *$ & $0.394 * *$ \\
\hline Line-33 & $3.905 * *$ & 0.221 & $81.149 * *$ & $1.493 * *$ & 0.893 & $0.633 * *$ & $0.644 * *$ \\
\hline Line-34 & $1.655 * *$ & 0.428 & $35.024 *$ & $-0.732 *$ & 0.808 & $-0.367 * *$ & $-0.356 * *$ \\
\hline Line-35 & $2.380 * *$ & $-2.516 * *$ & -26.176 & $-1.308 * *$ & -0.82 & $-0.867 * *$ & $-0.856 * *$ \\
\hline Line-36 & $1.105 * *$ & 0.311 & 25.699 & -0.157 & 0.023 & $1.133 * *$ & $-0.356 * *$ \\
\hline Line-37 & $1.780 * *$ & 0.459 & $38.074 * *$ & -0.608 & $-1.497 * *$ & $1.133 * *$ & $1.144 * *$ \\
\hline Line-38 & -0.22 & 0.351 & -1.501 & -0.352 & $-1.875 * *$ & $0.133 *$ & $-0.356 * *$ \\
\hline Line-39 & $7.605 * *$ & $2.434 * *$ & $183.250 * *$ & $1.098 * *$ & 0.01 & $0.133 *$ & $0.144 * *$ \\
\hline Line-40 & $-3.120 * *$ & $2.031 * *$ & -6.926 & -0.332 & -0.54 & $-0.867 * *$ & $-0.856 * *$ \\
\hline Line-41 & -0.795 & 0.259 & -9.826 & -0.133 & -0.857 & $0.633 * *$ & $0.644 * *$ \\
\hline Line-42 & $-0.970 *$ & $1.986 * *$ & $31.024 *$ & 0.047 & 0.84 & -0.117 & $0.144 * *$ \\
\hline Line-43 & -0.22 & 0.036 & -3.951 & $-0.857 *$ & $-1.395 *$ & $1.633 * *$ & $1.144 * *$ \\
\hline Line-44 & $5.305 * *$ & $3.204 * *$ & $188.450 * *$ & $-1.590 * *$ & -0.477 & $1.633 * *$ & $1.144 * *$ \\
\hline Line-45 & 0.43 & $1.471 * *$ & $37.649 * *$ & 0.293 & 0.073 & $1.133 * *$ & $1.144 * *$ \\
\hline Line-46 & $10.705 * *$ & 0.603 & $169.775 * *$ & 0.47 & $1.323 *$ & $1.133 * *$ & $0.644 * *$ \\
\hline Line-47 & $-0.970 *$ & $0.864 *$ & -1.851 & $1.443 * *$ & $-1.197 *$ & $-0.867 * *$ & $-0.856 * *$ \\
\hline CD@5\% & 0.807 & 0.807 & 26.768 & 0.724 & 1.089 & 0.132 & 0.059 \\
\hline \multicolumn{8}{|l|}{ Tester } \\
\hline $\mathbf{A A}$ & -0.249 & -2.303 & $-58.623 * *$ & $-0.221 * *$ & -0.218 & $-0.803 * *$ & $-0.793 * *$ \\
\hline IC & 0.249 & 2.303 & $58.623 * *$ & $0.221 * *$ & 0.218 & $0.803 * *$ & $0.793 * *$ \\
\hline CD@ $5 \%$ & 0.167 & 0.166 & 5.522 & 0.149 & 0.225 & 0.027 & 0.012 \\
\hline * Significa & \%; ** Signi & AA-Ar & IC- IC5508 & & & & \\
\hline
\end{tabular}


Table.3 Specific combining ability effects for earliness, yield, fruit quality and seed quality parameters in okra

\begin{tabular}{|c|c|c|c|c|c|c|c|}
\hline Crosses & $\begin{array}{l}\text { Number of fruits } \\
\text { per plant }\end{array}$ & $\begin{array}{l}\text { Average fruit } \\
\text { weight }(g)\end{array}$ & $\begin{array}{l}\text { Total fruit yield per } \\
\text { plant }(\mathrm{g})\end{array}$ & $\begin{array}{l}\text { Fruit length } \\
(\mathrm{cm})\end{array}$ & $\begin{array}{l}\text { Fruit diameter } \\
(\mathrm{mm})\end{array}$ & $\begin{array}{l}\text { No. of } \\
\text { ridges }\end{array}$ & $\begin{array}{l}\text { No. of } \\
\text { locules }\end{array}$ \\
\hline Line-1 $x$ AA & $1.048 *$ & -0.956 & 1.239 & $-1.129 *$ & -0.397 & $0.803 * *$ & $0.793 * *$ \\
\hline Line-1 x IC & $-1.048 *$ & 0.956 & -1.239 & $1.129 *$ & 0.397 & $-0.803 * *$ & $-0.793 * *$ \\
\hline Line-2 $x$ AA & $-3.152 * *$ & $2.774 * *$ & 22.066 & 0.543 & -1.407 & $0.303 * *$ & $0.293 * *$ \\
\hline Line-2 x IC & $3.152 * *$ & $-2.774 * *$ & -22.066 & -0.543 & 1.407 & $-0.303 * *$ & $-0.293 * *$ \\
\hline Line-3 x AA & $1.473 * *$ & $3.087 * *$ & $100.06 * *$ & $-2.254 * *$ & 0.99 & $-0.197 *$ & $-0.207 * *$ \\
\hline Line-3 x IC & $-1.473 * *$ & -3.087 & $-100.06 * *$ & $2.254 * *$ & -0.99 & $0.197 *$ & $0.207 * *$ \\
\hline Line-4 $x$ AA & $1.573 * *$ & -0.198 & 26.845 & $1.513 * *$ & 1.383 & $-0.197 *$ & $-1.207 * *$ \\
\hline Line-4 x IC & $-1.573 * *$ & 0.198 & -26.845 & $-1.513 * *$ & -1.383 & $0.197 *$ & $1.207 * *$ \\
\hline Line-5 x AA & $3.123 * *$ & $-1.318 *$ & 13.071 & -0.442 & -0.222 & $0.803 * *$ & $0.793 * *$ \\
\hline Line-5 x IC & $-3.123 * *$ & $1.318 *$ & -13.071 & 0.442 & 0.222 & $-0.803 * *$ & $-0.793 * *$ \\
\hline Line-6 $x$ AA & 0.423 & $-3.158 * *$ & $-63.691 * *$ & $-1.352 *$ & $-2.410 * *$ & $-0.697 * *$ & $-1.207 * *$ \\
\hline Line-6 x IC & -0.423 & $3.158 * *$ & $63.691 * *$ & $1.352 *$ & $2.410 * *$ & $0.697 * *$ & $1.207 * *$ \\
\hline Line-7 x AA & -0.402 & 0.099 & -4.143 & $-1.729 * *$ & -1.07 & $-0.697 * *$ & $-1.207 * *$ \\
\hline Line-7 x IC & 0.402 & -0.099 & 4.143 & $1.729 * *$ & 1.07 & $0.697 * *$ & $1.207 * *$ \\
\hline Line-8 $\times$ AA & 0.448 & $-1.351 *$ & -12.273 & 0.896 & -0.227 & $0.803 * *$ & $0.793 * *$ \\
\hline Line-8 x IC & -0.448 & $1.351 *$ & 12.273 & -0.896 & 0.227 & $-0.803 * *$ & $-0.793 * *$ \\
\hline Line-9 x AA & -0.202 & 1.052 & 27.705 & -0.429 & -1.132 & $0.803 * *$ & $0.293 * *$ \\
\hline Line-9 x IC & 0.202 & -1.052 & -27.705 & 0.429 & 1.132 & $-0.803 * *$ & $-0.293 * *$ \\
\hline Line-10 $\times$ AA & $1.473 * *$ & $-2.638 * *$ & -35.254 & $-2.229 * *$ & $-2.565 * *$ & $-1.197 * *$ & $-1.207 * *$ \\
\hline Line-10 x IC & $-1.473 * *$ & $2.638 * *$ & 35.254 & $2.229 * *$ & $2.565 * *$ & $1.197 * *$ & $1.207 * *$ \\
\hline Line-11 x AA & $-2.127 * *$ & $1.194 *$ & -6.718 & 0.246 & 1.393 & $0.803 * *$ & $0.793 * *$ \\
\hline Line-11 x IC & $2.127 * *$ & $-1.194 *$ & 6.718 & -0.246 & -1.393 & $-0.803 * *$ & $-0.793 * *$ \\
\hline
\end{tabular}




\begin{tabular}{|c|c|c|c|c|c|c|c|}
\hline Line-12 x AA & $1.948 * *$ & $1.289 *$ & $63.669 * *$ & $1.946 * *$ & 0.193 & $-0.197 *$ & $0.293 * *$ \\
\hline Line-12 x IC & $-1.948 * *$ & $-1.289 *$ & $-63.669 * *$ & $-1.946 * *$ & -0.193 & $0.197 *$ & $-0.293 * *$ \\
\hline Line-13 x AA & $1.523 * *$ & 0.692 & $45.351 *$ & -0.392 & $-1.865 *$ & $-0.197 *$ & $-0.707 * *$ \\
\hline Line-13 x IC & $-1.523 * *$ & -0.692 & $-45.351 *$ & 0.392 & $1.865 *$ & $0.197 *$ & $0.707 * *$ \\
\hline Line-14 x AA & $-1.327 *$ & -0.006 & -21.073 & $-1.554 * *$ & -0.175 & $-0.697 * *$ & $-0.207 * *$ \\
\hline Line-14 x IC & $1.327 *$ & 0.006 & 21.073 & $1.554 * *$ & 0.175 & $0.697 * *$ & $0.207 * *$ \\
\hline Line-15 x AA & -0.977 & 0.062 & -11.677 & $1.278 *$ & 1.208 & $0.803 * *$ & $0.793 * *$ \\
\hline Line-15 x IC & 0.977 & -0.062 & 11.677 & $-1.278 *$ & -1.208 & $-0.803 * *$ & $-0.793 * *$ \\
\hline Line-16 x AA & -0.052 & $2.267 * *$ & $57.261 * *$ & 0.038 & $1.873 *$ & $-0.197 *$ & $-0.207 * *$ \\
\hline Line-16 x IC & 0.052 & $-2.267 * *$ & $-57.261 * *$ & -0.038 & $-1.873 *$ & $0.197 *$ & $0.207 * *$ \\
\hline Line-17 x AA & $-2.902 * *$ & $3.942 * *$ & $50.504 * *$ & $-1.054 *$ & $3.143 * *$ & $-0.197 *$ & $-0.207 * *$ \\
\hline Line-17 x IC & $2.902 * *$ & $-3.942 * *$ & $-50.504 * *$ & $1.054 *$ & $-3.143 * *$ & $0.197 *$ & $0.207 * *$ \\
\hline Line-18 x AA & $-1.402 * *$ & -2.766 & $107.632 * *$ & $2.783 * *$ & -1.402 & $0.803 * *$ & $0.793 * *$ \\
\hline Line-18 x IC & $1.402 * *$ & $2.766 * *$ & $107.632 * *$ & $-2.783 * *$ & 1.402 & $-0.803 * *$ & $-0.793 * *$ \\
\hline Line-19 x AA & $1.173 *$ & 0.714 & $38.376 *$ & $1.171 *$ & -0.337 & $0.303 * *$ & $0.293 * *$ \\
\hline Line-19 x IC & $-1.173 *$ & -0.714 & $-38.376 *$ & $-1.171 *$ & 0.337 & $-0.303 * *$ & $-0.293 * *$ \\
\hline Line-20 $x$ AA & 0.048 & 0.229 & 12.416 & $2.946 * *$ & $1.965 *$ & $0.803 * *$ & $0.793 * *$ \\
\hline Line-20 x IC & -0.048 & -0.229 & -12.416 & $-2.946 * *$ & $-1.965 *$ & $-0.803 * *$ & $-0.793 * *$ \\
\hline Line-21 x AA & $-2.577 * *$ & 0.712 & -21.926 & 0.721 & -0.477 & $0.803 * *$ & $0.793 * *$ \\
\hline Line-21 x IC & $2.577 * *$ & -0.712 & 21.926 & -0.721 & 0.477 & $-0.803 * *$ & $-0.793 * *$ \\
\hline Line-22 x AA & $-3.952 * *$ & 0.347 & $-99.409 * *$ & $-1.154 *$ & -0.747 & $0.803 * *$ & $0.793 * *$ \\
\hline Line-22 x IC & $3.952 * *$ & -0.347 & $99.409 * *$ & $1.154 *$ & 0.747 & $-0.803 * *$ & $-0.793 * *$ \\
\hline Line-23 x AA & $-4.777 * *$ & -3.121 & $-176.180 * *$ & 0.396 & $-2.367 * *$ & $0.803 * *$ & $0.793 * *$ \\
\hline Line-23 x IC & $4.777 * *$ & $3.121 * *$ & $176.180 * *$ & -0.396 & $2.367 * *$ & $-0.803 * *$ & $-0.793 * *$ \\
\hline Line-24 x AA & $5.773 * *$ & $2.709 * *$ & $201.496 * *$ & $-1.092 *$ & -1.342 & $-1.197 * *$ & $-1.207 * *$ \\
\hline
\end{tabular}


Int.J.Curr.Microbiol.App.Sci (2018) 7(7): 1879-1888

\begin{tabular}{|c|c|c|c|c|c|c|c|}
\hline Line-24 x IC & $-5.773 * *$ & -2.709 & $-201.496 * *$ & $1.092 *$ & 1.342 & $1.197 * *$ & $1.207 * *$ \\
\hline Line-25 x AA & $-1.702 * *$ & $3.107 * *$ & $53.757 * *$ & 0.816 & 1.488 & $0.803 * *$ & $0.793 * *$ \\
\hline Line-25 x IC & $1.702 * *$ & $-3.107 * *$ & $-53.757 * *$ & -0.816 & -1.488 & $-0.803 * *$ & $-0.793 * *$ \\
\hline Line-26 x AA & $-3.577 * *$ & $-1.826 * *$ & $-122.264 * *$ & $-1.074 *$ & -1.137 & $0.303 * *$ & $-0.207 * *$ \\
\hline Line-26 x IC & $3.577 * *$ & $1.826 * *$ & $122.264 * *$ & $1.074 *$ & 1.137 & $-0.303 * *$ & $0.207 * *$ \\
\hline Line-27 x AA & $2.798 * *$ & -0.723 & 31.744 & $1.146 *$ & $1.990 *$ & $-0.697 * *$ & $-0.707 * *$ \\
\hline Line-27 x IC & $-2.798 * *$ & 0.723 & -31.744 & $-1.146 *$ & $-1.990 *$ & $0.697 * *$ & $0.707 * *$ \\
\hline Line-28 x AA & -0.977 & $-1.781 * *$ & $-61.116 * *$ & $1.296 *$ & -0.465 & $0.803 * *$ & $0.793 * *$ \\
\hline Line-28 x IC & 0.977 & $1.781 * *$ & $61.116 * *$ & $-1.296 *$ & 0.465 & $-0.803 * *$ & $-0.793 * *$ \\
\hline Line-29 x AA & 0.548 & $3.609 * *$ & $95.706 * *$ & 0.721 & 0.303 & $-1.197 * *$ & $-1.207 * *$ \\
\hline Line-29 x IC & -0.548 & $-3.609 * *$ & $-95.706 * *$ & -0.721 & -0.303 & $1.197 * *$ & $1.207 * *$ \\
\hline Line-30 x AA & $-2.452 * *$ & -0.108 & $-47.514 *$ & -0.849 & 0.418 & $0.803 * *$ & $0.793 * *$ \\
\hline Line-30 x IC & $2.452 * *$ & 0.108 & $47.514 *$ & 0.849 & -0.418 & $-0.803 * *$ & $-0.793 * *$ \\
\hline Line-31 x AA & $-1.577 * *$ & $-2.388 * *$ & $-101.264 * *$ & -0.754 & -0.37 & $-0.197 *$ & $-0.207 * *$ \\
\hline Line-31 x IC & $1.577 * *$ & $2.388 * *$ & $101.264 * *$ & 0.754 & 0.37 & $0.197 *$ & $0.207 * *$ \\
\hline Line-32 x AA & $-1.552 * *$ & $1.922 * *$ & 24.868 & 0.761 & $1.928 *$ & $-0.197 *$ & $-0.457 * *$ \\
\hline Line-32 x IC & $1.552 * *$ & $-1.922 * *$ & -24.868 & -0.761 & $-1.928 *$ & $0.197 *$ & $0.457 * *$ \\
\hline Line-33 x AA & $-2.002 * *$ & $-1.318 *$ & $-99.403 * *$ & 0.096 & $1.853 *$ & $2.303 * *$ & $2.293 * *$ \\
\hline Line-33 x IC & $2.002 * *$ & $1.318 *$ & $99.403 * *$ & -0.096 & $-1.853 *$ & $-2.303 * *$ & $-2.293 * *$ \\
\hline Line-34 x AA & $1.048 *$ & $-1.682 * *$ & -33.561 & $1.871 * *$ & 0.008 & $0.303 * *$ & $0.293 * *$ \\
\hline Line-34 x IC & $-1.048 *$ & $1.682 * *$ & 33.561 & $-1.871 * *$ & -0.008 & $-0.303 * *$ & $-0.293 * *$ \\
\hline Line-35 x AA & $-3.277 * *$ & 0.349 & $-55.314 * *$ & $3.046 * *$ & -0.885 & $0.803 * *$ & $0.793 * *$ \\
\hline Line-35 x IC & $3.277 * *$ & -0.349 & $55.314 * *$ & $-3.046 * *$ & 0.885 & $-0.803 * *$ & $-0.793 * *$ \\
\hline Line-36 x AA & -0.952 & 0.672 & -1.803 & $-1.504 * *$ & -1.207 & $-1.197 * *$ & $0.293 * *$ \\
\hline Line-36 x IC & 0.952 & -0.672 & 1.803 & $1.504 * *$ & 1.207 & $1.197 * *$ & $-0.293 * *$ \\
\hline
\end{tabular}




\begin{tabular}{|c|c|c|c|c|c|c|c|}
\hline Line-37 x AA & $1.273 *$ & $-1.176 *$ & -16.033 & $-1.454 * *$ & 0.253 & $-1.197 * *$ & $-1.207 * *$ \\
\hline Line-37 x IC & $-1.273 *$ & $1.176 *$ & 16.033 & $1.454 * *$ & -0.253 & $1.197 * *$ & $1.207 * *$ \\
\hline Line-38 x AA & $3.173 * *$ & 0.092 & $54.895 * *$ & -0.359 & 0.93 & $-0.197 *$ & $0.293 * *$ \\
\hline Line-38 x IC & $-3.173 * *$ & -0.092 & $-54.895 * *$ & 0.359 & -0.93 & $0.197 *$ & $-0.293 * *$ \\
\hline Line-39 x AA & $4.198 * *$ & -0.851 & $113.151 * *$ & $-1.949 * *$ & 1.05 & $-0.197 *$ & $-0.207 * *$ \\
\hline Line-39 x IC & $-4.198 * *$ & 0.851 & $-113.151 * *$ & $1.949 * *$ & -1.05 & $0.197 *$ & $0.207 * *$ \\
\hline Line-40 x AA & $1.123 *$ & -0.228 & 20.916 & -0.679 & 1.195 & $0.803 * *$ & $0.793 * *$ \\
\hline Line-40 x IC & $-1.123 *$ & 0.228 & -20.916 & 0.679 & -1.195 & $-0.803 * *$ & $-0.793 * *$ \\
\hline Line-41 x AA & $1.898 * *$ & 0.739 & $50.667 * *$ & $1.621 * *$ & 0.073 & $-0.697 * *$ & $-0.707 * *$ \\
\hline Line-41 $x$ IC & $-1.898 * *$ & -0.739 & $-50.667 * *$ & $-1.621 * *$ & -0.073 & $0.697 * *$ & $0.707 * *$ \\
\hline Line-42 $\times$ AA & 0.823 & $-2.498 * *$ & $-47.746 *$ & 0.991 & -1.135 & 0.053 & $-0.207 * *$ \\
\hline Line-42 $\times$ IC & -0.823 & $2.498 * *$ & $47.746 *$ & -0.991 & 1.135 & -0.053 & $0.207 * *$ \\
\hline Line-43 x AA & 0.423 & $3.427 * *$ & $95.809 * *$ & -0.504 & -0.64 & $-1.697 * *$ & $-1.207 * *$ \\
\hline Line-43 x IC & -0.423 & $-3.427 * *$ & $-95.809 * *$ & 0.504 & 0.64 & $1.697 * *$ & $1.207 * *$ \\
\hline Line-44 x AA & $-2.002 * *$ & $1.444 *$ & -6.888 & 0.013 & 0.893 & $-1.697 * *$ & $-1.207 * *$ \\
\hline Line-44 x IC & $2.002 * *$ & $-1.444 *$ & 6.888 & -0.013 & -0.893 & $1.697 * *$ & $1.207 * *$ \\
\hline Line-45 x AA & $3.073 * *$ & -0.788 & 31.438 & $-1.904 * *$ & -0.037 & $-1.197 * *$ & $-1.207 * *$ \\
\hline Line-45 x IC & $-3.073 * *$ & 0.788 & -31.438 & $1.904 * *$ & 0.037 & $1.197 * *$ & $1.207 * *$ \\
\hline Line-46 x AA & $1.648 * *$ & $-3.407 * *$ & $-56.437 * *$ & 0.193 & 0.043 & $-1.197 * *$ & $-0.707 * *$ \\
\hline Line-46 x IC & $-1.648 * *$ & $3.407 * *$ & $56.437 * *$ & -0.193 & -0.043 & $1.197 * *$ & $0.707 * *$ \\
\hline Line-47 x AA & $1.873 * *$ & $-2.246 * *$ & -23.699 & $-1.204 *$ & -0.557 & $0.803 * *$ & $0.793 * *$ \\
\hline Line-47 x IC & $-1.873 * *$ & $2.246 * *$ & 23.699 & $1.204 *$ & 0.557 & $-0.803 * *$ & $-0.793 * *$ \\
\hline CD@ $9 \%$ & 1.024 & 1.141 & 37.854 & 1.025 & 1.541 & 0.186 & 0.083 \\
\hline
\end{tabular}


Sca involving $\mathrm{H} \times \mathrm{H}$ combination could be used in developing of the varieties to exploit additive gene action by pedigree. Whereas, the crosses involving the combination of $\mathrm{L} \mathrm{x}$ $\mathrm{L}$ could be used for exploitation of heterosis by recurrent selection.

In conclusion, these promising crosses were identified as overall high combiners and these could be utilized for development of elite breeding population by allowing through mixing them to achieve new genetic recombination and then subjecting the resultant population to recurrent selection.

\section{References}

Anonymous, 2014, Indian Horticultural Database, 2014. http://www.nhb.gov. in.

Ashwani, K., Baranwal, D. K., Aparna, J. and Srivastava, K., 2013, Combining Ability and Heterosis for yield and its contributing characters in okra (Abelmoschus esculentus (L.) Moench). Madras Agric. J. 100 (1-3): 30-35.

Hazem, A. O., Eldekashy, M. H. Z. and Helaly, A. A. 2013, Combining ability and heterosis studies for yield and its components in some cultivars of okra (Abelmoschus esculentus (L.) Moench). American-Eurasian J. Agric. Environ. Sci. 13(2): 162-167.
Jasol, F. S., 1989, Export prospectus of horticultural produce from India. Indian Hort. 34: 79-85.

Kempthorne, O., 1957, An introduction to genetic statistics. John Wiley and Sons, New York, pp. 408-711.

Khatik, K. R., Chaudhary, R. and Khatik, C. L., 2012, Combining ability effects for yield and its component in okra (Abelmoschus esculentus (L.) Moench). Ann. Hort. 5(2): 240-245.

Kumar, N. K. L., and Kumar, S. G., 2015, Heterosis and combining ability for grain yield and its component traits of newly developed inbred lines of maize (Zea mays L.). Green farming. 6(3): 452-456.

Laxman, M., Shanthakumar, G., Thimmanna, P. O., Udaykumar, K., Prakash, G. and Sateesh, A., 2013, Nutritional enhancement for iron content and combining ability studies in newly derived inbred lines of okra (Abelmoschus esculentus Moench L.). Molecular Plant Breed. 4(3) 24-30.

Nagesh, G. C. Mulge, R., Rathod, V., Basavaraj, B. and Mahaveer, S. M.. 2014, Heterosis and combining ability studies in okra (Abelmoschus esculentus (L.) Moench) for yield and Quality parameters. Bioscon. 9(4): 1717-1723.

\section{How to cite this article:}

Harshavardhan Gowda, V., Shantappa Tirakannanavar, R.C. Jagadeesha, V.D. Gasti, S.M. Veeresha and Ashok. 2018. Combining Ability for Yield and Quality Traits in Early Generation Inbred Lines of Okra. Int.J.Curr.Microbiol.App.Sci. 7(07): 1879-1888. doi: https://doi.org/10.20546/ijcmas.2018.707.223 